\title{
COMPARISON OF ROBUST METHODS FOR ARMA ORDER ESTIMATION
}

\author{
SAMUEL FLIMMEL, MATEJ ČAMAJ, IVANA MALÁ, JIŘÍ PROCHÁZKA \\ University of Economics, Prague, Faculty of Informatics and Statistics, \\ Department of Statistics and Probability, \\ W. Churchill Sq. 1938/4, Prague, Czech Republic \\ email: samuel.flimmel@vse.cz,matej.camaj@vse.cz,malai@vse.cz,xproj16@vse.cz
}

\begin{abstract}
In financial markets, we are facing big data problems. Loads of information are stored almost every second, but usually standard methods have problems to process them all. With growing number of observations, the probability of outlier presence also rises. That is the reason of increase in importance to work with sufficiently robust methods. As it is known, standard methods are not able to work correctly with outliers and consequently standard estimates are usually biased. ARMA processes are frequently used in financial mathematics and one of the important steps is to estimate the order of a given process. Usually it is the second step in Box-Jenkins method after solving stationarity and seasonality. In this paper we present robust methods for ARMA order estimating and we compare them using a simulation study. For the simulation study we are using the $R$ statistical software.
\end{abstract}

Key words: robust methods, ARMA process, ARMA order estimation

JEL Codes: C02, C22, G10

\section{DOI: 10.15611/amse.2017.20.10}

\section{Introduction}

Autoregressive moving-average (ARMA) processes are well known and widely used in the financial world. They are one of the basic econometric tools for modeling time series. To estimate an ARMA process from time series data, firstly it is necessary to solve stationarity and seasonality of the given time series, what is often done by decomposing the process. Another important step is to determine the order of the ARMA process. After the order determination, we can estimate the process parameters themselves.

However, due to extensive exploiting of big data nowadays, we face problems related to an increased probability of outlier presence. The outliers complicate the ARMA process estimation because they can cause an estimator to be biased. There are several robust methods for ARMA order estimation that take into account outlier presence. For example Maronna et al. (2006), Rousseeuw and Croux (1992), and others suggest different approaches that should help with the problem. Naturally, every method has certain advantages as well as disadvantages. We choose four of these approaches, and after their brief introduction, we compare them by performing a simulation study.

In Section 2, we establish the notation that we work within this paper. In Section 3, we introduce two basic outlier models: the additive outlier model and the innovative outlier model. In Section 4, we give a brief introduction to the robust methods that we work with. In Section 5, we show results of the simulation study used to compare the methods. 


\section{Definitions and notation}

Let us define white noise, which is a zero mean mutually uncorrelated time series $\left\{\varepsilon_{n}, n \in N_{0}\right\}$ with an unknown constant variance $\sigma_{\varepsilon}^{2}>0$.

We define an autoregressive process $\operatorname{AR}(p)$ by the equation:

$$
X_{n}=\varphi_{1} X_{n-1}+\varphi_{2} X_{n-2}+\ldots+\varphi_{p} X_{n-p}+\varepsilon_{n},
$$

where $\varphi_{1}, \varphi_{2}, \ldots, \varphi_{p} \in R$ are parameters, $\left\{\varepsilon_{n}, n \in N_{0}\right\}$ is white noise and $\varphi_{p} \neq 0$.

We define a moving-average process $\operatorname{MA}(q)$ by the equation:

$$
X_{n}=\varepsilon_{n}+\theta_{1} \varepsilon_{n-1}+\theta_{2} \varepsilon_{n-2}+\ldots+\theta_{q} \varepsilon_{n-q},
$$

where $\theta_{1}, \theta_{2}, \ldots, \theta_{q} \in R$ are parameters, $\left\{\varepsilon_{n}, n \in N_{0}\right\}$ is white noise and $\theta_{q} \neq 0$.

We define an autoregressive-moving-average process $\operatorname{ARMA}(p, q)$ by the equation:

$$
X_{n}=\varphi_{1} X_{n-1}+\ldots+\varphi_{p} X_{n-p}+\varepsilon_{n}+\theta_{1} \varepsilon_{n-1}+\ldots+\theta_{q} \varepsilon_{n-q},
$$

where $\varphi_{1}, \ldots, \varphi_{p}, \theta_{1}, \ldots, \theta_{q} \in R$ are the parameters, $\left\{\varepsilon_{n}, n \in N_{0}\right\}$ is white noise and coefficients $\varphi_{p}, \theta_{q} \neq 0$.

We define an autocovariance function of lag $k R(k)$ of stationary process $\left\{X_{n}, n \in N_{0}\right\}$ as:

$$
R(k)=E\left(X_{k}-\mu\right)\left(X_{0}-\mu\right),
$$

where $\mu$ is the expected value of the process.

We define an autocorrelation function (ACF) of lag $k$ of stationary process $\left\{X_{n}, n \in N_{0}\right\}$ as:

where $\sigma_{X}^{2}$ is the variance of the process.

$$
\rho(k)=\frac{R(k)}{\sigma_{X}^{2}},
$$

Let us define a partial autocorrelation function (PACF) of lag $k$ of stationary process $\left\{X_{n}, n \in N_{0}\right\}$ as:

$$
\alpha(k)= \begin{cases}\rho(1), & k=1, \\ \operatorname{corr}\left(X_{k}-\widetilde{X_{k}}, X_{0}-\widetilde{X_{0}}\right), & k>1,\end{cases}
$$

where corr is a function for correlation and $\widetilde{X_{0}}$, respectively $\widetilde{X_{k}}$, is a projection of $X_{0}$, respectively $X_{k}$, onto the Hilbert's space spanned by $X_{1}, X_{2}, X_{3}, \ldots, X_{k-1}$.

\section{Outlier models}

There are several models for simulating outliers in a time series (e.g. Maronna et al., 2006). Let us introduce two of them: the additive outlier model and the innovative outlier model.

\subsection{Additive outliers}

The additive outlier (AO) model was originally introduced by Fox (1972). In the AO model, we assume that we do not observe the process of our interest $\left\{X_{n}, n \in N_{0}\right\}$, but we actually observe a process $\left\{Y_{n}, n \in N_{0}\right\}$ defined as:

$$
Y_{n}=X_{n}+Z_{n}
$$


where processes $\left\{X_{n}, n \in N_{0}\right\}$ and $\left\{Z_{n}, n \in N_{0}\right\}$ are assumed to be independent of one another.

Let $\left\{Z_{n}, n \in N_{0}\right\}$ be a process with independent and identically distributed random variables that have a normal mixture distribution with a degenerate central component:

$$
Z_{n} \sim(1-\beta) \delta_{0}+\beta N\left(\mu_{Z}, \sigma_{Z}^{2}\right),
$$

where $\delta_{0}$ is the point mass distribution located at zero, and we assume that the normal component $N\left(\mu_{Z}, \sigma_{Z}^{2}\right)$ has a variance significantly higher than the process $\left\{X_{n}, n \in N_{0}\right\}, \sigma_{Z}^{2} \gg$ $\sigma_{X}^{2}$

The probability of an outlier occurrence is represented by $\beta$, which is usually small. Consequently, the probability of an occurrence of 2 outliers in a row is a much smaller $\beta^{2}$, which means that the AO model generates mostly isolated outliers.

Figure 1: Example of an additive outlier.

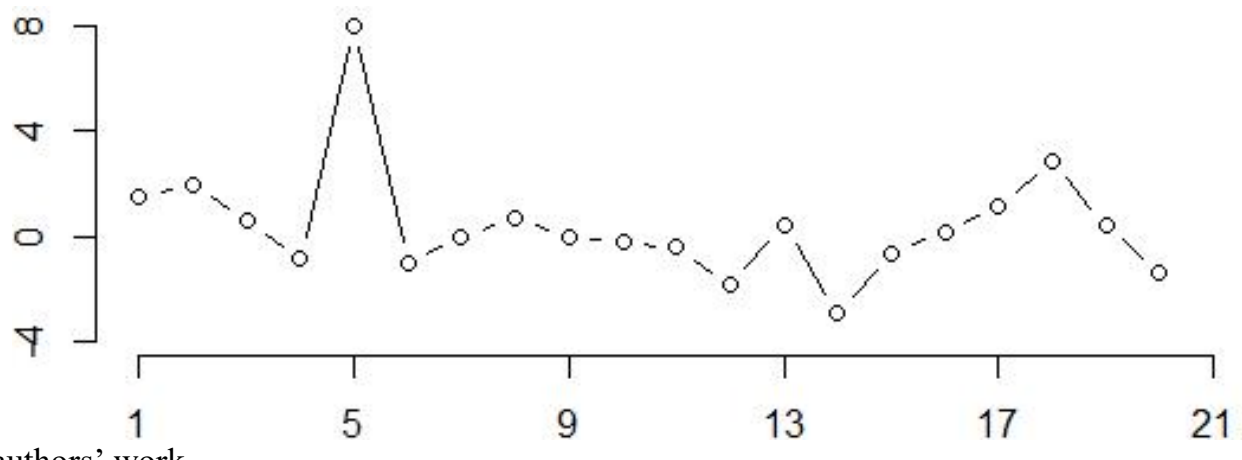

Source: The authors' work

\subsection{Innovative outliers}

The innovative outlier (IO) model was originally introduced also by Fox (1972), who used the term "type II outliers". The IO model works with a highly specialized form of outliers that can occur in a linear processes such as $\operatorname{AR}(p), \operatorname{ARMA}(p, q)$ or $\operatorname{ARIMA}(p, d, q)$.

Figure 2: Example of an innovative outlier.

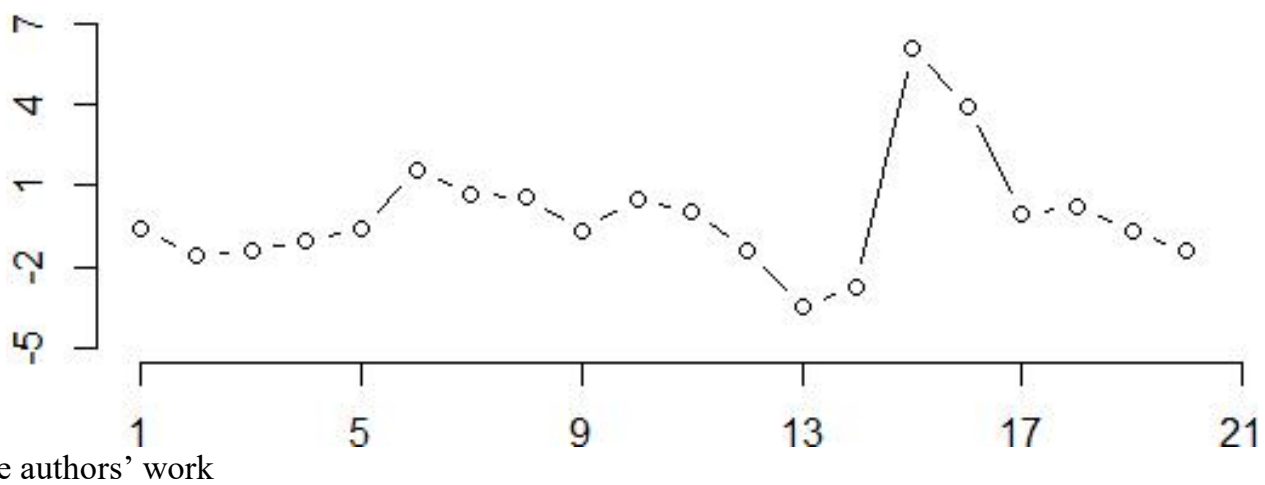

Source: The authors' work

For simplicity, we introduce only a special case that we use for this paper. The white noise process $\left\{\varepsilon_{n}, n \in N_{0}\right\}$ from the definitions of $\operatorname{AR}(p), \operatorname{MA}(q)$ or $\operatorname{ARMA}(p, q)$ is sometimes also called the innovations process. The IO model generates outliers directly in the innovations process. For the IO model, we assume independent identically distributed (i.i.d.) random variables in the process $\left\{\tau_{n}, n \in N_{0}\right\}$ with a normal mixture distribution

$$
\tau_{n} \sim(1-\beta) N\left(0, \sigma_{\tau}^{2}\right)+\beta N\left(0, \sigma_{I}^{2}\right),
$$


where $\sigma_{I}^{2} \gg \sigma_{\tau}^{2}$. The IO outlier affects not only the current observation but also subsequent observations.

\section{Robust methods}

There is only a limited amount of robust methods for ARMA order estimates. Principally, we only need to estimate the autocorrelation function of the process because there is a theorem expressing the relation between the autocorrelation function $\rho(l)$ and the partial autocorrelation function $\alpha(k)$ (e.g. Yafee, 2000):

$$
\alpha(k)=\frac{\left|\begin{array}{ccccc}
1 & \rho(1) & \cdots & \rho(k-2) & \rho(1) \\
\rho(1) & 1 & \cdots & \rho(k-3) & \rho(2) \\
\vdots & \vdots & \ddots & \vdots & \vdots \\
\rho(k-1) & \rho(k-2) & \cdots & \rho(1) & \rho(k)
\end{array}\right|}{\left|\begin{array}{cccc}
1 & \rho(1) & \cdots & \rho(k-1) \\
\rho(1) & 1 & \cdots & \rho(k-2) \\
\vdots & \vdots & \ddots & \vdots \\
\rho(k-1) & \rho(k-2) & \cdots & 1
\end{array}\right|}, k>1,
$$

where $|\cdot|$ represents determinant. Having the ACF and the PACF of the process, we can estimate orders of the $\operatorname{ARMA}(p, q)$ process.

We introduce 4 methods, which we briefly describe, and we compare them by means of a simulation study. For an overview of robust methods for ACF estimates, you can see Dürre et al. (2015).

\subsection{Method based on median correlation}

The method based on median correlation was introduced by Chakhchoukh (2010). This method is quite intuitive because instead of using the mean, we work only with the median from equation (4). The median is well known as a robust estimator of location. Firstly we centre our observations $X_{0}, X_{1}, \ldots, X_{m}$ by:

$$
\widetilde{X}_{k}=X_{k}-\operatorname{med}\left(X_{0}, X_{1}, \ldots, X_{m}\right),
$$

where $\operatorname{med}(\cdot)$ gives the median of the observations.

Then we estimate the ACF using:

$$
\widehat{\rho}_{\text {med }}(k)=\frac{\operatorname{med}\left(\widetilde{X}_{0}, \widetilde{X}_{1}, \ldots, \widetilde{X}_{m}\right)}{\operatorname{med}\left(\widetilde{X}_{0}{ }^{2}, \widetilde{X}_{1}{ }^{2}, \ldots, \widetilde{X}_{m}{ }^{2}\right)} .
$$

For a consistent estimation of $\rho(k)$, a nonlinear transformation of $\widehat{\rho}_{\text {med }}(k)$, which has to be determined numerically, is necessary (Dürre et al., 2015). This nonlinear transformation can be based on a Monte Carlo simulation.

\subsection{Method based on trimming}

The method based on trimming is described in several papers (e.g. Dürre et al., 2015). This method is based on omitting some terms in the calculation of the standard ACF. Firstly we estimate an autocovariance function: 
where

$$
\widehat{R}_{\text {trim }}^{(\tau)}(k)=\frac{1}{\sum_{i=0}^{m-k} L_{i}^{(\tau)} L_{i+k}^{(\tau)}}\left(\sum_{i=0}^{m-k}\left(X_{i}-\bar{X}^{(\tau)}\right)\left(X_{i+k}-\bar{X}^{(\tau)}\right) L_{i}^{(\tau)} L_{i+k}^{(\tau)}\right)
$$

$$
\bar{X}^{(\tau)}=\frac{1}{\sum_{i=0}^{m} L_{i}^{(\tau)}} \sum_{i=0}^{m} X_{i} L_{i}^{(\tau)} \text { and } L_{i}^{(\tau)}= \begin{cases}1, & X_{t}<X_{i}<X_{m-t} \\ 0, & \text { else }\end{cases}
$$

with $t=\lfloor\tau(m+1)\rfloor-1$ for some $0 \leq \tau<0.5$. Chan and Wei (1992) proposed $0.01 \leq \tau \leq 0.1$, depending on the suspected percentage of outliers.

The ACF estimator $\hat{\rho}_{\text {trim }}^{(\tau)}(k)$ is calculated as the ratio of trimmed autocovariance and trimmed variance $\widehat{R}_{\text {trim }}^{(\tau)}(0)$. Similarly, as for $\widehat{\rho}_{\text {med }}(k)$, a nonlinear transformation is necessary to obtain a consistent estimation of $\rho(k)$. In addition, $\widehat{\rho}_{\text {med }}(k)$ is a limiting case of $\widehat{\rho}_{\text {trim }}^{(\tau)}(k) \underset{\tau \rightarrow 0.5}{\longrightarrow} \widehat{\rho}_{\text {med }}(k)$.

\subsection{Method based on the Gnanadesikan-Kettenring approach}

The method based on the Gnanadesikan-Kettenring approach, named after the researchers who introduced it (Gnanadesikan \& Kettenring, 1972), exploits an idea that can be formulated as:

$$
R(k)=\frac{1}{4}\left(\operatorname{var}\left(X_{0}+X_{k}\right)-\operatorname{var}\left(X_{0}-X_{k}\right)\right) .
$$

This method is also called a scale approach. Equation (15) is written here already in a simplified form. For a more general formula, you can see e.g. Huber (1981).

In the context of scale estimation, Rousseeuw and Croux (1992) proposed a robust estimator $\mathrm{Q}_{\mathrm{m}}$ :

$$
Q_{m}=c\left[\left|X_{i}-X_{j}\right|, i<j\right]_{k},
$$

where $c$ is a factor included for consistency, at the Gaussian distribution $c=2.2191$ and $[\cdot]_{k}$ is $k$-th order statistic and $k$ is defined as:

$$
k=\left\lfloor\frac{\left(\begin{array}{l}
m \\
2
\end{array}\right)+2}{4}\right\rfloor+1
$$

where function $[\cdot]$ denotes the integer part and $m$ is the number of observations.

Using the formulas above, we get an estimator:

$$
\widehat{\rho}_{G K}(k)=\frac{Q_{m-k}^{2}(u+v)-Q_{m-k}^{2}(u-v)}{Q_{m-k}^{2}(u+v)+Q_{m-k}^{2}(u-v)},
$$

where $u$ is the vector $\left(X_{m-k}, X_{m-k+1}, \ldots, X_{m}\right)$ and $v$ is the vector $\left(X_{0}, X_{1}, \ldots, X_{k}\right)$. The method of this robust ACF estimator was presented by Ma and Genton (2000). 


\subsection{Method based on robust filtering}

The method based on robust filtering was described by Maronna et al. (2006). This approach takes the time series structure into account. The idea is to have robustly filtered values instead of the original observation and to calculate ACF from these filtered values. Principally, we replace outliers by some reasonable values.

Firstly, we estimate the order of the AR process which we use for robust filtering. It can be done by a robust AIC criterion that was proposed also by Maronna et al. (2006). Or we can use a "long" AR process instead.

Secondly, we obtain fitted values using the robustly filtered $\tau$-scale estimate. Finally, we calculate autocorrelation function.

\section{Simulation study}

The simulation study was designed in the software $R$ (R Core Team, 2013) and we use the $\mathrm{R}$ package robts (see Dürre, 2016). However, the package was still not approved by CRAN at the time of the study, so certain functions were coded by the authors of this paper to validate the correctness of the package. After the validation was succesfull, we used functions from the package to obtain estimations in the simulation study.

To determine whether an autocorrelation function of the order $k$ is still significant, we use Bartlett's approximation (Bartlett, 1946):

$$
\widehat{\rho}(k) \sim N\left(0, \frac{1+2 \sum_{i=1}^{k_{0}} \widehat{\rho}(i)^{2}}{m}\right), k>k_{0},
$$

if $\rho(k)=0$ for $k>k_{0}$. Therefore, we search for a $k_{0}$ that holds:

$$
|\widehat{\rho}(k)| \geq 2 \sqrt{\frac{1+2 \sum_{i=1}^{k_{0}} \widehat{\rho}(i)^{2}}{m}}, k>k_{0} .
$$

Similarly, for partial autocorrelation function, we use Quenouille's approximation (Quenouille, 1949):

$$
|\widehat{\alpha}(k)| \geq 2 \sqrt{\frac{1}{m}}, k>k_{0} .
$$

ARMA process is known as a process without the $k_{0}$ in (20) and (21). There always is some $k_{0}$ (for stationary ARMA process) that will hold both inequalities (21) and (22), but long orders are not preferable from the practical point of view. We choose maximum value of $k_{0}$ equal to 6 . If there is no $k_{0} \leq 6$, we assume there exists no $k_{0}$ at all.

For every examined case, we run 1000 simulations and have 1000 observations. The percentage of outliers present in a single simulation is chosen randomly with an uniform distribution, i.e. $\omega \sim U([0.00,0.05])$.

All 4 described methods are applied to each simulation as either an AR process with the order $p$ between 1 and 6, an MA process with the order $q$ between 1 and 6 , or a general ARMA process. In the case of the simulations for the AR process, we put results of the MA processes of all orders into a single category. Analogously, for the simulations for the MA process, we put results of all AR processes into a single category. The simulations are evaluated according the rules mentioned above. 


\subsection{Autoregressive process $\mathrm{AR}(3)$}

Absolute values of the parameters of the AR(3) process are generated randomly with an uniform distribution, i.e. $\varphi_{i} \sim U((0.2,1.0))$. Values of $\varphi_{i}$ being close to zero are not taken into account because they are difficult to observe. The sign of the parameters is generated randomly with Bernoulli's distribution with the probability of success $p=0.5$. Subsequently, we check whether these parameters lead to a stationary process and we repeat the procedure until it is necessary.

Results for the AO model $\left(\sigma_{Z}=10\right)$ can be seen in Table 1 .

Table 1: Process AR(3) with data contaminated by the AO model.

\begin{tabular}{ccccc}
\hline Model & Median & Trim & GK & Filter \\
\hline AR(1) & $0.0 \%$ & $0.0 \%$ & $0.0 \%$ & $0.0 \%$ \\
AR(2) & $0.0 \%$ & $0.2 \%$ & $0.0 \%$ & $0.0 \%$ \\
AR(3) & $2.6 \%$ & $0.2 \%$ & $30.3 \%$ & $70.2 \%$ \\
AR(4) & $3.5 \%$ & $0.9 \%$ & $8.7 \%$ & $8.1 \%$ \\
AR(5) & $7.7 \%$ & $1.7 \%$ & $10.9 \%$ & $8.8 \%$ \\
AR(6) & $11.3 \%$ & $5.4 \%$ & $15.2 \%$ & $6.1 \%$ \\
MA & $20.5 \%$ & $20.8 \%$ & $9.0 \%$ & $2.8 \%$ \\
ARMA & $54.4 \%$ & $70.8 \%$ & $25.9 \%$ & $4.0 \%$ \\
\hline
\end{tabular}

Source: The authors' work

We can see that the method based on trimming is the worst. More than two thirds of the simulations are considered as ARMA process. As the second worst, we can consider the method based on median. At least $2.6 \%$ of the simulations are evaluated correctly, but this percentage should be much higher. There is a notable difference between these two simple methods and the method based on GK approach. Almost one third of the simulations are evaluated correctly, and another third of the simulations are considered to be AR processes. Clearly, the best method is the one based on robust filtering (section 4.4). More than two thirds of the simulations are evaluated correctly and only $6.8 \%$ of the simulations are not considered as an AR process.

Figure 3: Process AR(3) with data contaminated by the AO model.

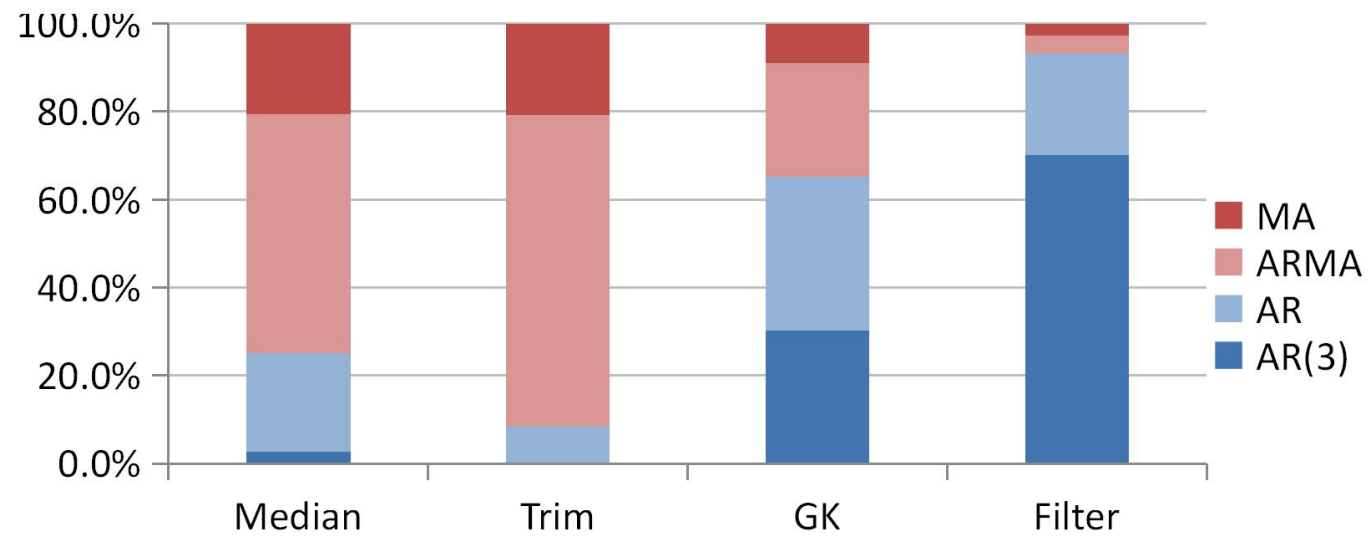

Source: The authors' work

Results for the IO model $\left(\sigma_{I}=10\right)$ can be seen in Table 2 .

If we take into account only simulations with $\omega<0.01$, which means only less than $1 \%$ contamination, then the results will not be much different. It means that the methods are 
robust enough because they are not much affected by outliers. On the other hand, the results are not as good as we would expect. Satisfying results are given only by the robust filtering method and possibly by the method based on GK approach.

We can see the results also in a graphical form in Figure 3 where we put all AR processes except the correct one $\mathrm{AR}(3)$ into a single category.

Table 2: AR(3) process with data contaminated by the IO model.

\begin{tabular}{ccccc}
\hline Model & Median & Trim & GK & Filter \\
\hline AR(1) & $0.0 \%$ & $0.0 \%$ & $0.0 \%$ & $0.0 \%$ \\
AR(2) & $0.0 \%$ & $0.1 \%$ & $0.1 \%$ & $0.0 \%$ \\
AR(3) & $1.9 \%$ & $1.0 \%$ & $12.9 \%$ & $32.9 \%$ \\
AR(4) & $2.6 \%$ & $1.2 \%$ & $6.3 \%$ & $10.7 \%$ \\
AR(5) & $4.7 \%$ & $1.7 \%$ & $8.1 \%$ & $22.6 \%$ \\
AR(6) & $11.4 \%$ & $4.1 \%$ & $14.1 \%$ & $22.2 \%$ \\
MA & $20.0 \%$ & $18.8 \%$ & $13.3 \%$ & $3.8 \%$ \\
ARMA & $59.4 \%$ & $73.1 \%$ & $45.2 \%$ & $7.8 \%$ \\
\hline
\end{tabular}

Source: The authors' work

We can see that all the methods give worse results in comparison to the additive outlier model. This is caused by the fact that the innovative outliers are more difficult to process. The method based on trimming is the worst again, and only $1 \%$ of the simulations are evaluated correctly. Almost $2 \%$ of the simulations are evaluated correctly for the method based on median and only $30 \%$ of the simulations are considered as AR processes. The method based on GK approach provides correct identification for $12.9 \%$ of the simulations, but almost $60 \%$ of the simulations mislead to MA or ARMA. The method based on robust filtering evaluates one third of the simulations correctly as AR(3), meanwhile only $11.6 \%$ of the simulations are not evaluated as AR process.

In Figure 4 we can see the results in a graphical form.

Figure 4: AR(3) process with data contaminated by the IO model.

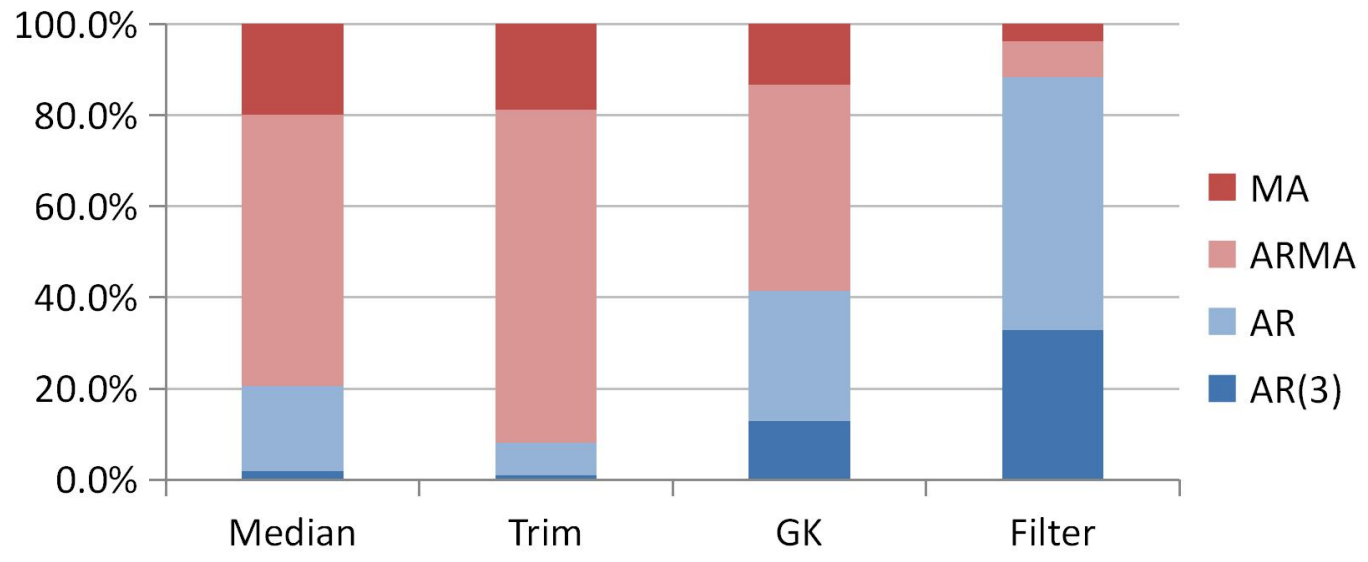

Source: The authors' work

In Table 3 , only simulations with percentage of contamination less than $1 \%$ are shown. We can see that the results are similar to those shown in Table 1. It means that even a quite small portion of outliers can affect the estimation. This is caused by the fact that innovative outliers affect not an only observation but the next several observations as well. 
Table 3: AR(3) process with data contaminated by the IO model with $\varepsilon<0.01$.

\begin{tabular}{ccccc}
\hline Model & Median & Trim & GK & Filter \\
\hline AR(1) & $0.0 \%$ & $0.0 \%$ & $0.0 \%$ & $0.0 \%$ \\
AR(2) & $0.0 \%$ & $0.5 \%$ & $0.0 \%$ & $0.0 \%$ \\
AR(3) & $1.5 \%$ & $0.5 \%$ & $25.1 \%$ & $63.1 \%$ \\
AR(4) & $2.6 \%$ & $1.5 \%$ & $6.7 \%$ & $11.8 \%$ \\
AR(5) & $3.6 \%$ & $3.1 \%$ & $8.7 \%$ & $10.3 \%$ \\
AR(6) & $12.8 \%$ & $5.1 \%$ & $14.4 \%$ & $8.7 \%$ \\
MA & $24.1 \%$ & $21.5 \%$ & $11.3 \%$ & $3.6 \%$ \\
ARMA & $55.4 \%$ & $67.7 \%$ & $33.8 \%$ & $2.6 \%$ \\
\hline
\end{tabular}

Source: The authors' work

\subsection{Moving-average process $\mathrm{MA}(4)$}

Similarly as for the $\mathrm{AR}(3)$ process, absolute values of the parameters of the MA(4) process are generated randomly with an uniform distribution, i.e. $\theta_{i} \sim U((0.2,1.0))$. Values of $\theta_{i}$ being close to zero are not taken into account because they are difficult to observe. The sign of the parameters is generated randomly with Bernoulli's distribution with the probability of success $p=0.5$.

Results for the AO model $\left(\sigma_{Z}=10\right)$ can be seen in Table 4 .

Table 4: MA(4) process with data contaminated by the AO model.

\begin{tabular}{ccccc}
\hline Model & Median & Trim & GK & Filter \\
\hline MA(1) & $0.1 \%$ & $0.0 \%$ & $0.0 \%$ & $0.0 \%$ \\
MA(2) & $0.5 \%$ & $0.0 \%$ & $0.2 \%$ & $0.3 \%$ \\
MA(3) & $4.9 \%$ & $2.2 \%$ & $4.3 \%$ & $4.3 \%$ \\
MA(4) & $56.1 \%$ & $21.0 \%$ & $76.6 \%$ & $77.7 \%$ \\
MA(5) & $8.8 \%$ & $15.4 \%$ & $5.4 \%$ & $4.7 \%$ \\
MA(6) & $10.1 \%$ & $20.0 \%$ & $3.0 \%$ & $3.1 \%$ \\
AR & $9.9 \%$ & $9.5 \%$ & $7.4 \%$ & $7.1 \%$ \\
ARMA & $9.6 \%$ & $31.9 \%$ & $3.1 \%$ & $2.8 \%$ \\
\hline
\end{tabular}

Source: The authors' work

Figure 5: MA(4) process with data contaminated by the AO model.

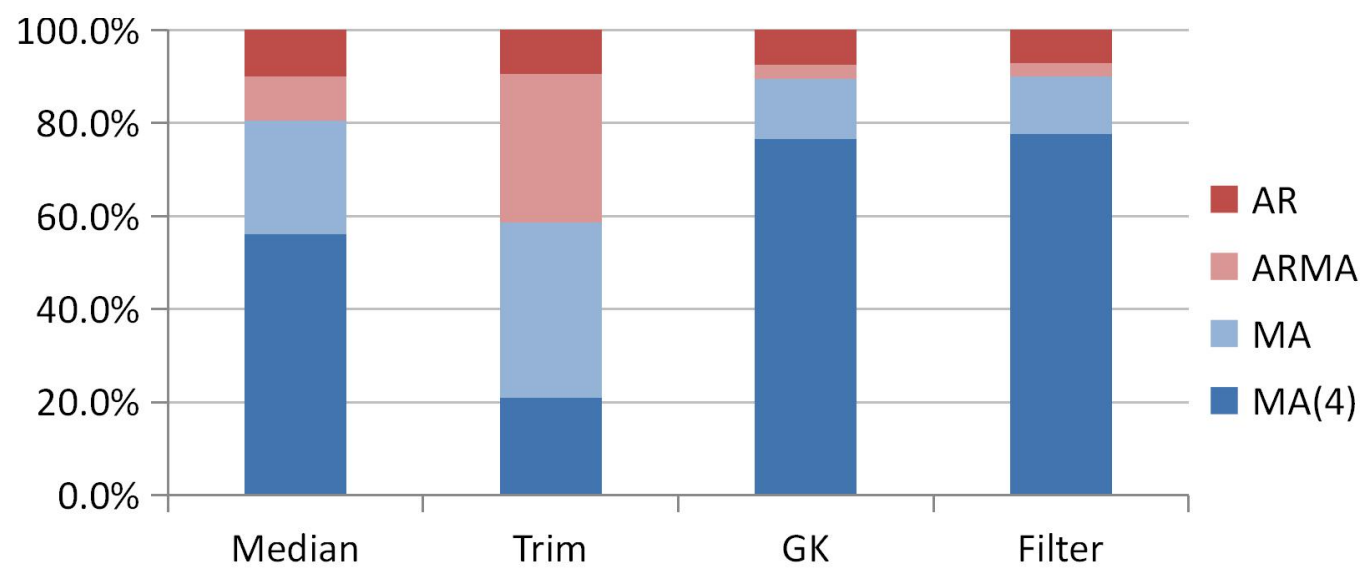

Source: The authors' work 
The results are markedly better than for the $\mathrm{AR}(3)$ process with additive outliers. The worst result are observed again after using the method based on trimming, but the percentage of correct estimates is $21 \%$ (only $0.2 \%$ for the AR(3) process with the AO model). Much better results are obtained with the method based on median. More than a half of the simulations are evaluated correctly, that is $56.1 \%$. Only $19.5 \%$ of the simulations are not evaluated as MA processes. The remaining two methods perform similarly well, both of them yield correct evaluations for approximately $77 \%$ of the simulations. Only approximately $10 \%$ of the simulations are not evaluated as MA processes.

In Figure 5 we can see the results in a graphical form. We put the MA processes of all orders into a single category, except for the correct MA(4) process.

Results for the IO model $\left(\sigma_{I}=10\right)$ can be seen in Table 5 .

Table 5: MA(4) process with data contaminated by the IO model.

\begin{tabular}{ccccc}
\hline Model & Median & Trim & GK & Filter \\
\hline MA(1) & $0.0 \%$ & $0.0 \%$ & $0.0 \%$ & $0.0 \%$ \\
MA(2) & $0.3 \%$ & $0.3 \%$ & $0.0 \%$ & $0.2 \%$ \\
MA(3) & $4.0 \%$ & $1.4 \%$ & $4.5 \%$ & $5.7 \%$ \\
MA(4) & $60.7 \%$ & $22.1 \%$ & $80.0 \%$ & $75.9 \%$ \\
MA(5) & $8.6 \%$ & $15.9 \%$ & $3.2 \%$ & $3.6 \%$ \\
MA(6) & $7.8 \%$ & $19.4 \%$ & $2.5 \%$ & $2.9 \%$ \\
AR & $9.2 \%$ & $8.5 \%$ & $6.3 \%$ & $8.7 \%$ \\
ARMA & $9.4 \%$ & $32.4 \%$ & $3.5 \%$ & $3.0 \%$ \\
\hline
\end{tabular}

Source: The authors' work

Figure 6: MA(4) process with data contaminated by the IO model.

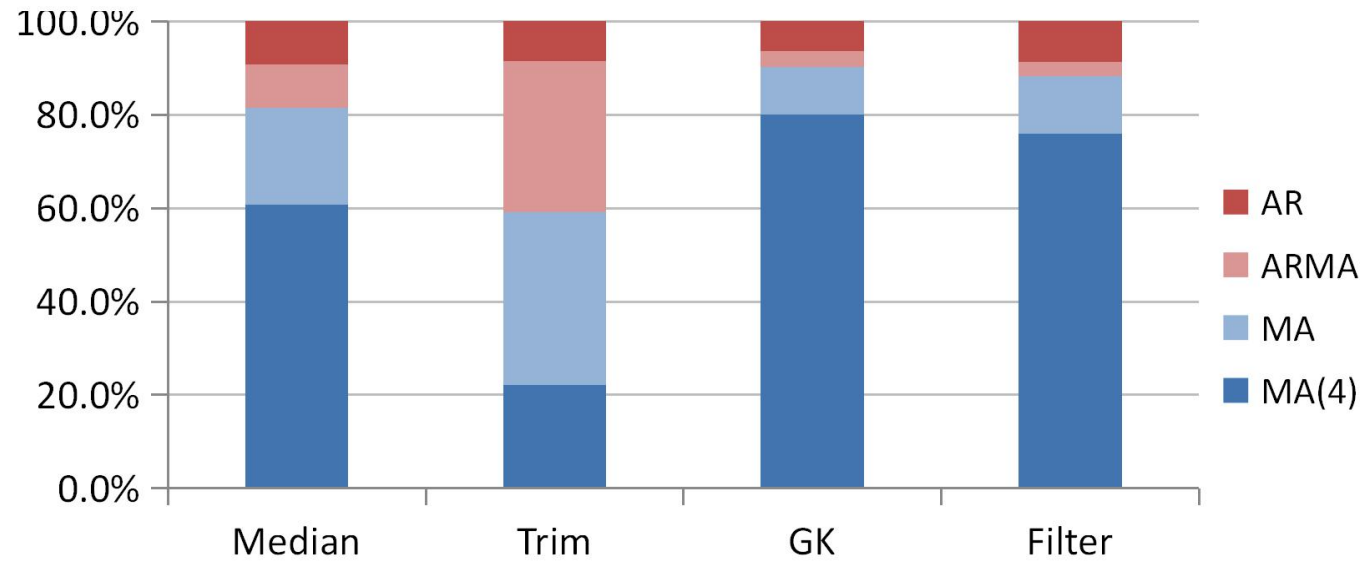

Source: The authors' work

We can see in Table 5 that innovative outliers are not so effective in the case of the MA process. The results are much better in comparison with Table 2. As always, the worst results are given by the method based on trimming. This method leads to correct determination in $22.1 \%$ of the simulations. The method based on median provides correct determination in $60.7 \%$, which is the best result for this method in our simulation study. Only less than $20 \%$ of the simulations are not evaluated as MA processes. The method based on robust filtering is not the best for the first time in this simulation study, but it still leads to correct identification almost in $76 \%$ of the simulations. The best method is the one based on GK approach in this 
case. It enables correct identification in $80 \%$ of the simulations and only less than $10 \%$ of the simulations are not evaluated as MA process. In Figure 6 we can see the results in a graphical form.

\section{Conclusion}

We introduced two models for outliers. The additive outlier model generates mostly isolated outliers. On the contrary, the innovative outlier model affects not only current observation but also subsequent observations.

We introduced four robust methods for ACF estimation. Every robust method is based on a different idea which we briefly described. When we have ACF robust estimators, we can robustly estimate PACF, too. Subsequently we are able to estimate orders of the ARMA process.

We performed a simulation study in which we compared the four chosen methods. We noticed that the most difficult case for the assessment is the AR process with innovative outliers. For this process, the results were the worst with every method. On the other hand, the innovative outliers do not affect the MA process so distinctively.

The method based on trimming led to the worst results. In the case of the AR processes, it does not provide almost any correct identification. For the MA process, the results were better, but the correct determination rate of approximately $20 \%$ is still insufficient.

The median-based method led to slightly better results, however, for the AR processes, the evaluation was correct only in $2 \%$ of the simulations. In the case of the MA processes, we reached the level of almost $60 \%$ of successful determinations, what can be considered as a quite positive result.

The two simple methods, the results of which are summarized above, were markedly worse in comparison to more sophisticated methods. One of the sophisticated method we used is based on the GK approach. It led to $30 \%$ of correct identification in the case of the AR process with additive outliers, respectively to $12 \%$ of correct identification in the case of the AR process with innovative outliers. For the MA processes, it yielded results similar to the best method, and in the case of the MA process with innovative outliers, it was actually the best method with $80 \%$ of success in the identification.

Overall, the best results were obtained by using the method based on robust filtering. In three out of four cases, it provided the highest successful identification rate, in the fourth case it was very close to the best result. Using this method, more than $70 \%$ of identifications were successful every time, except for the AR process with innovative outliers. For this special case, the evaluations were correct for approximately $33 \%$ of the simulations.

In order to conclude, based on the presented results of the performed simulation study, we consider the method based on robust filtering as the most appropriate to estimate orders of ARMA processes.

\section{Acknowledgements}

This paper is supported by the grant F4/17/2017 (Robustnost' v úmyselnom useknutí časového radu) which has been provided by the Internal grant agency of the University of Economics in Prague.

\section{References}

[1] Bartlett, M. S. 1946. On the Theoretical Specification and Sampling Properties of Autocorrelated Time-Series. Supplement to the Journal of the Royal Statistical Society, vol. 8, iss. 1, pp. 27-41. 
[2] Dürre, A., Fried, R., Liboschik, T. 2015. Robust estimation of (partial) autocorrelation. Wiley Interdisciplinary Reviews: Computational Statistics, vol. 7, iss. 3, pp. 205-222.

[3] Dürre, A et. al. 2016. robts: Robust Time Series Analysis. R package version 0.3.0/r220. http://R-Forge.R-project.org/projects/robts/

[4] Fox, A.J. 1972. Outliers in time series. Journal of the Royal Society, vol. 34, iss. 3, pp. 350-363.

[5] Gnanadesikan, R., Kettenring, J. R. 1972. Robust estimates, residuals, and outlier detection with multiresponse data. Biometrics, vol. 28, pp. 81-124.

[6] Huber, P. J. 1981. Robust Statistics. New York: Wiley.

[7] Chakhchoukh, Y. 2010. A new robust estimation method for ARMA models. IEEE Transactions on Signal Processing, vol. 58, iss. 7, pp. 3512-3522.

[8] Chan, W.-S., Wei, W. W. 1992. A comparison of some estimators of time series autocorrelations. Computational statistics \& data analysis, vol. 14, iss. 2, pp. 149-163.

[9] Ma, Y. and Genton M. 2000. Highly robust estimation of the autocovariance function, Journal of time series analysis, vol. 21, iss. 6, pp. 663-684.

[10] Maronna, R., Martin, D., Yohai, V. 2006. Robust Statistics: Theory and Methods. Chichester: John Wiley \& Sons, Ltd. ISBN 978-0-470-01092-1.

[11] Quenouille, M.H., 1949. Approximate tests of correlation in time-series. Journal of the Royal Statistical Society: Series B, vol. 11, iss. 3, pp. 68-84.

[12] R Core Team 2013: R: a language and environment for statistical computing. Vienna: $\mathrm{R}$ Foundation for Statistical Computing, http://www.r-project.org/.

[13] Rousseeuw, P. J., Croux, C. 1992. Explicit scale estimators with high breakdown point. L1-Statistical Analyses and Related Methods, pp. 77-92.

[14] Yaffee, R., McGee, M. 2000. An Introduction to Time Series Analysis and Forecasting. Academic Press. ISBN 9780127678702. 